\title{
Thermomechanical tensile properties of deposited Inconel 718 superalloy over a wide range of strain rate and temperature
}

\author{
Martina Scapin ${ }^{1}$, Lorenzo Peroni ${ }^{1}$, Kangbo Yuan $^{2}$ and Weiguo Guo $^{2}$ \\ ${ }^{1}$ Politecnico di Torino, Department of Mechanical and Aerospace Engineering, Corso Duca degli \\ Abruzzi 24, 10129, Turin, Italy \\ ${ }^{2}$ School of Aeronautics, Northwestern Polytechnical University, Xi'an, 710072, China
}

\begin{abstract}
Nickel-based superalloys show high strength retained also at high temperature and they are widespread used for structural components exposed during services to high temperature combined with high strain rate or impact loading conditions. The objective of this study was the investigation of the plastic flow behaviour of Laser Metal Deposited Nickelbased superalloy Inconel718. The material was manufactured at Northwestern Polytechnical University in China. Specimens with three different heat treatment conditions were investigated: as-deposited, directly aged and aged after homogenization and solution. High strain rate tensile tests were performed on the direct Hopkinson bar setup developed at DYNLab laboratory at Politecnico di Torino. At a nominal strain rate of $1500 \mathrm{~s}^{-1}$ the temperature sensitivity was investigated between 20 and 1000 ${ }^{\circ} \mathrm{C}$. An induction heating system was adopted, and the temperature was monitored by thermocouples and infrared pyrometer and high-speed camera. The results showed the materials strength decreases as a function of temperature with a significant drop starting from $800{ }^{\circ} \mathrm{C}$. An asymmetric tension-compression behaviour was found by comparing the results with data in compression. The strain rate influence was investigated at room temperature and very limited or negligible sensitivity was found covering six orders of magnitude in strain rate.
\end{abstract}

\section{Introduction}

Nickel-based superalloys show high strength retained also at high temperature joined with high corrosion resistance. These aspects promote the widespread use of this class of material for manufacturing structural components which could be exposed to high temperature during services (even higher than $800{ }^{\circ} \mathrm{C}$ [1]) or harsh environments. Nickel-based superalloys are well suited for several applications in different fields, among which are aerospace, aviation, marine, nuclear, chemical, and power generation industries. Limiting the applications to the aerospace industry, the Nickel-based alloys are widely used to manufacture aeroengine parts [2], for which also the resistance to impact loads is an important property to be considered. In case of impact, high-rate deformation and fracture occurs. The previous considerations highlight the need of understanding the mechanical response in case of combined high 
temperature and high strain rate conditions for a safe design and realization of highperformance engine parts.

The components made of Nickel-based superalloys when manufactured by conventional machining methods might have high costs, usually long delivery time and a high wasting ratio material [3-5]. A good alternative solution is represented by the metal Additive Manufacturing (AM) technology which can be employed for the fabrication and for the repair of components, with the addition of the important advantage of being able also to renovate their geometry. The main disadvantage when as-deposited components are considered is related to the poor mechanical properties resulting from a completely different microstructure with respect to that obtained with traditional manufacturing methods. Hence, an important aspect is represented by the choice of an appropriate heat treatment to improve microstructure and mechanical properties.

The objective of this article is to investigate the tensile plastic flow behaviour of Inconel 718 superalloy produced by Laser Metal Deposition (LMD) as a function of the heat treatment to which different microstructure states correspond. A wide range in temperature was investigated at high strain-rate while in quasi-static condition only room temperature tests were performed.

\section{$2 \quad$ Material and specimens preparation}

The Inconel 718 dog-bone specimens were obtained starting from bars manufactured by LMD in the State Key Laboratory of Solidification Processing at Northwestern Polytechnical University (MPU) in China [6]. The tensile tests were performed such that the loading direction was the deposition direction (i.e., direction of height increase during manufacturing). Three different heat treatment conditions were investigated: as-deposited (referred to $\mathrm{aD}$, which means without heat treatment), direct-aged (referred to DA) and aged after homogenization \& solution (referred to HT). A detailed description of the processes is reported in [7]. The aim of the heat treatments was to improve the microstructure by maximizing the presence of strengthening phases [2-4]. In fact, due to rapid prototyping, high power input and lack of mechanical pressure during the LMD, the printed material, which is expected to take the place of the forged counterpart, usually features coarse columnar crystals, elements micro-segregation, anisotropy, and initial defects in microstructures. With the purpose to predict the expected materials strength, preliminary microstructure analyses were performed on the specimens in the different heat treatment conditions. This allowed the identification of the most important features affecting the mechanical properties in LMD Inconel 718. The analyses mainly evaluated the dimension and structure of the grains and the presence of strengthening phases $\gamma^{\prime}$ and $\gamma^{\prime \prime}$ [7]. The latter represents the main strengthening precipitates in Inconel 718, since it has a Body-Centered Cubic (BCC) structure, compared with Face-Centered Cubic (FCC) one of the phase $\gamma^{\prime}$. The microstructural results showed that the as-deposited microstructure was anisotropic and characterized by large columnar grains containing a small percentage in volume of $\gamma^{\prime \prime}$ particles. The direct aging was not sufficient to completely solute the dendrite structure, preserving the anisotropic microstructure, even if a high amount of strengthening phases precipitates. In specimens after homogenization, solution and aging small-size grains were observed and similarly as in the direct-aged specimens, a great amount of strengthening precipitates was observed.

\section{Experimental procedure}

The plastic flow behaviour of Inconel 718 was investigated by performing tensile tests on cylindrical dog-bone specimens with a gage section diameter of $3 \mathrm{~mm}$ and a gage length of 
$5 \mathrm{~mm}$. The same geometry was used both for quasi-static and high strain rate tests to not introduce geometrical effects and make possible a direct comparison of the results.

Quasi-static and high strain rate tests were carried out at DYNLab laboratory of Politecnico di Torino in Italy. The nominal strain rates were 0.001 and $1500 \mathrm{~s}^{-1}$ respectively. At high strain rate, a wide range of temperatures was investigated starting from room temperature up to $1000{ }^{\circ} \mathrm{C}$ : the testing temperatures were decided to be able to well appreciate the material behaviour as expected from the results obtained for compression tests in [7] on the same materials.

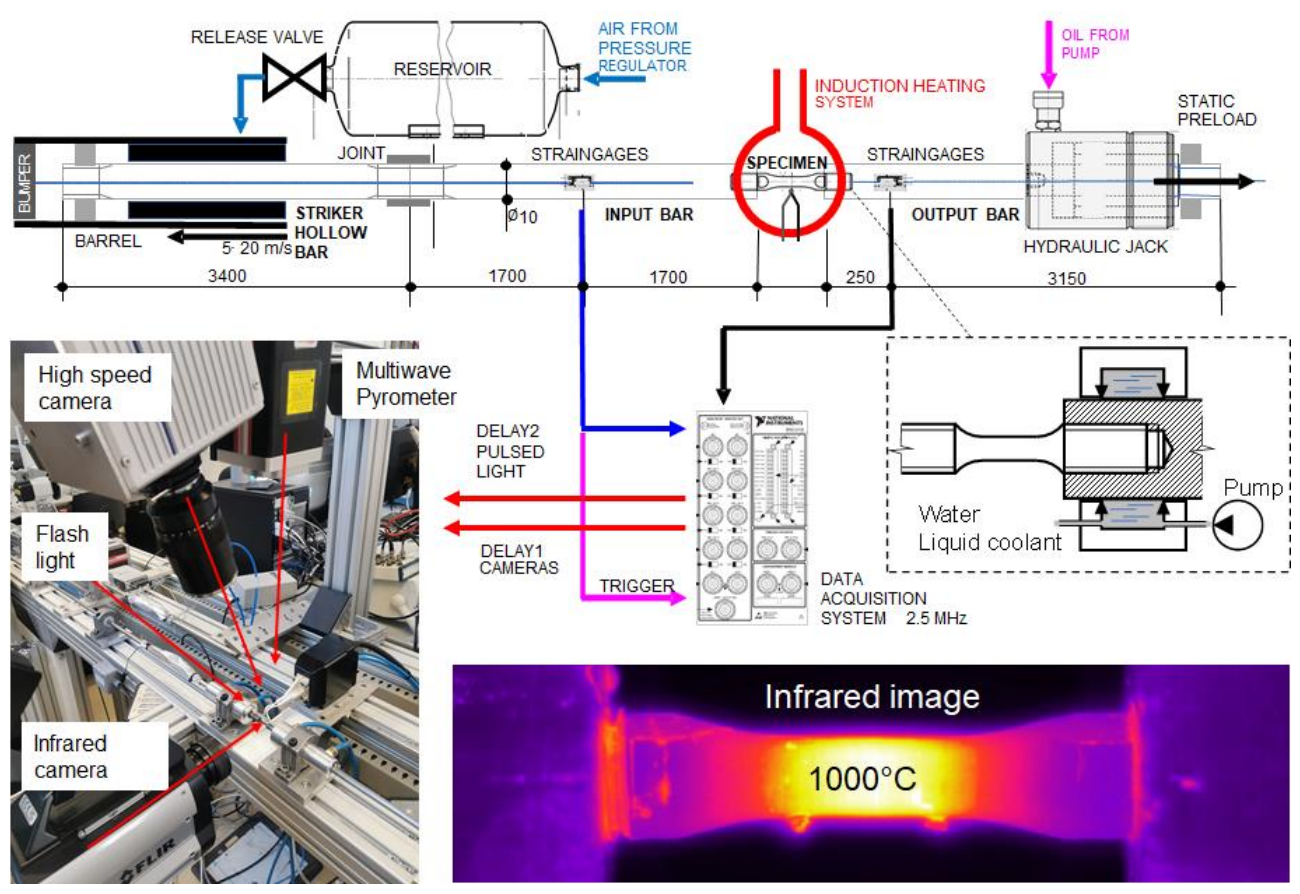

Fig. 1. Hopkinson Bar setup at DYNLab laboratory at Politecnico di Torino.

The quasi-static tests at room temperature were performed by means of the standard electromechanical testing machine Zwick Z100 by imposing a testing speed of $0.005 \mathrm{~mm} / \mathrm{s}$. The high strain rate tests were performed on a Hopkinson Bar setup in the direct configuration (see Figure 1). A detailed description of the setup is reported in [8-9]. A hollow striker bar is launched by compressed air and impacts an anvil at one end of the input bar generating the incident wave. During the testing campaign the level of pressure of the compressed air was adjusted as a function of the expected material strength to maintain the nominal strain rate stable. On input and output bars semiconductor strain-gages were adopted which have a high gain factor but require a calibration procedure for the non-linear behaviour. A static preload was imposed to guarantee the perfect alignment and the absence of internal clearances.

The heating of the specimens was obtained by using an induction heating system. Each specimen was heated to the target temperature and then held 5 minutes at this temperature before the test, as performed in [7] on the same materials for compression tests. The heating of the bars was limited using water-cooled supports, as indicated in the scheme of Fig. 1. 

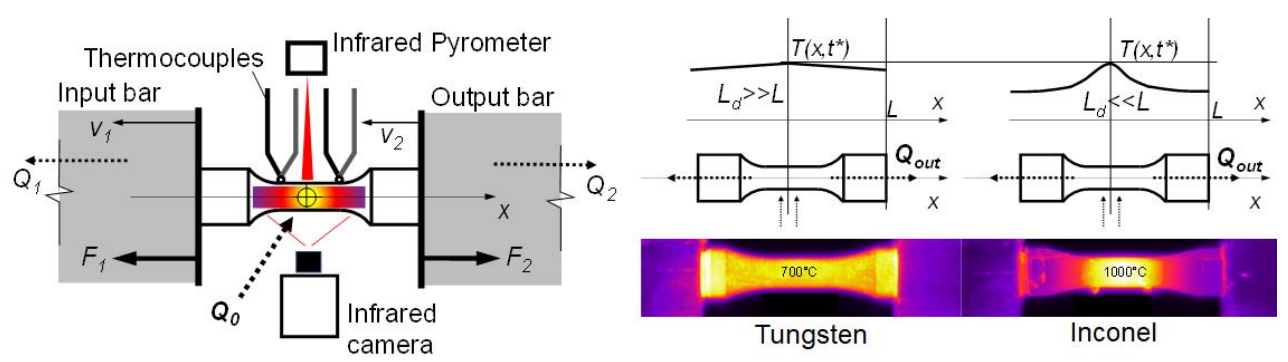

Fig. 2. Scheme of the temperature control systems adopted in dynamic high temperature tests (left); comparison between heating obtained on pure tungsten and Inconel 718 cylindrical dog-bone specimens with the same dimension by imposing the same heating power.

Three different systems to control the temperature of the specimens were adopted, as reported in Fig. 2. Two K-type thermocouples were welded directly on the specimen surface at the end of the gage length. Two multiwave infrared pyrometers were used to measure the maximum temperature reached in the gage length (Williamson Pro MW-10-20-C in the range of temperatures $475-1750^{\circ} \mathrm{C}$ and Williamson Pro MW-20-5-C in the range of temperatures $150-475^{\circ} \mathrm{C}$ ). Finally, an infrared camera (FLIR X6900SC) was used to check the uniformity of the temperature over the specimen. Each of them has pros and cons [10]. A summary of them is here reported. The reliability of the measurement performed by the thermocouples depends on the welding and can be affected by the electromagnetic field. In addition, they are too slow to appreciate the temperature increment due to adiabatic heating. The measurement coming from the infrared camera depends on the emissivity of the material which needs to be calibrated. Moreover, it depends on the temperature itself, hence it can vary during a dynamic test because of the adiabatic heating. In this study a high-speed infrared camera was adopted, which allowed also to obtain the information on the adiabatic heating during the tests. The multiwave infrared pyrometers can give the temperature measurement without knowing the emissivity of the material, but the spot over which they measure the temperature is bigger than the sample, so the signal strength has to be adjusted to be able to perform the measure, but this procedure reduces the range of measurable temperature. By taking into account these considerations, the authors decided to use all of these measuring systems and to check the consistency between the performed measurements. The thermal diffusivity of Inconel is low which corresponds to a short characteristic width defined as $l_{d}=2(\alpha \mathrm{t})^{0,5}$. At each time, $l_{d}$ gives the distance at which the amplitude of the heat flux reduces $e$ times with respect to the value it has where the high temperature is imposed. When the characteristic width is much shorter than the characteristic system size, the temperature can differ drastically from one part of the sample to another one. Gradients can be very large, and temperature is highly time-dependent. A large increase in temperature localized in one region of the sample will not be able to induce the same temperature increment also in far regions if the time is short enough that the diffusion length remains small. The thermal inertia of the specimen depends on the thermal diffusivity $\alpha$ : in a material with high thermal diffusivity, the heat moves rapidly through it because the material conducts the heat quickly relative to its volumetric heat capacity or 'thermal bulk'. This means that in such a material the uniform condition is easy to be reached but the maximum achievable temperature is low by fixing the heating power. The opposite happens in case of materials with low thermal diffusivity. This is in accordance with the temperature distribution reported in Fig. 2, in which the heat distribution obtained in a test performed at $1000{ }^{\circ} \mathrm{C}$ on Inconel 718 was compared to that obtained during a test performed at $700^{\circ} \mathrm{C}$ on pure tungsten, which has a higher thermal diffusivity. 


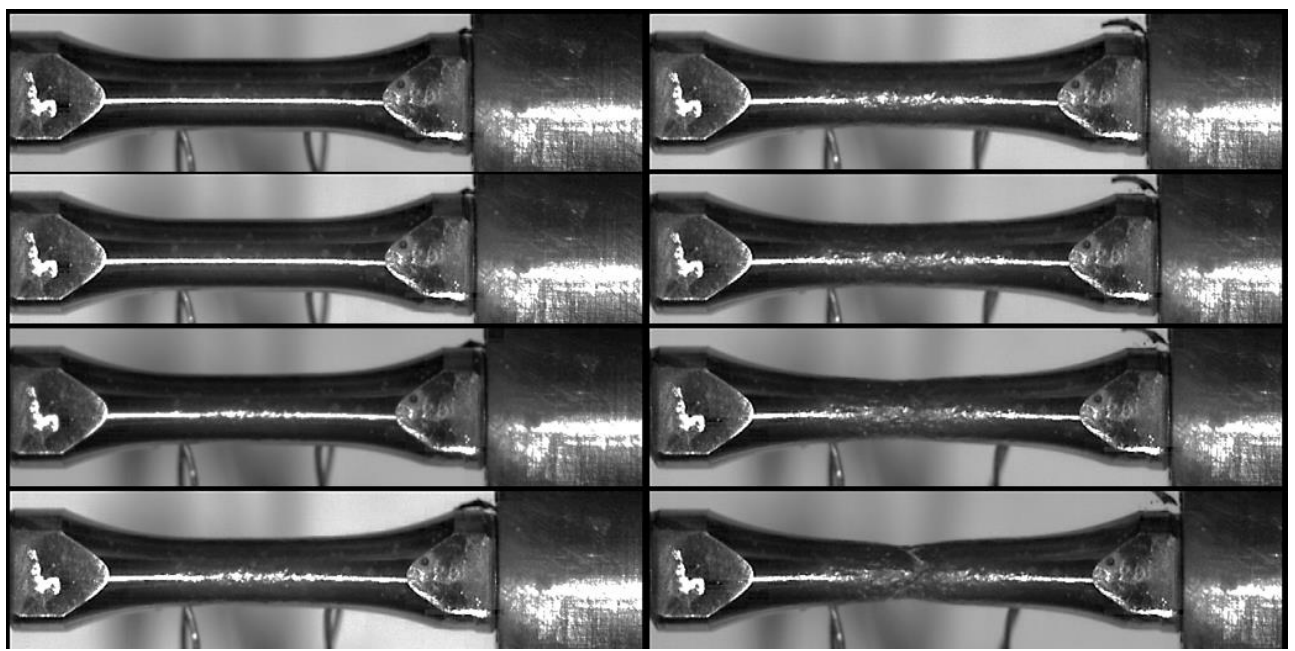

Fig. 3. Sequence of images of a high strain rate test at $850{ }^{\circ} \mathrm{C}$ on $\mathrm{HT}$ specimen: resolution of $52 \times 128$ pixels, framerate of $100000 \mathrm{fps}$, shutter of $1 / 100000 \mathrm{~s}$, time interval between the reported images of 50 microseconds.

Each test was also video recorded to obtain the deformation of the specimen directly measured on the sample by tracking the motion of some points with the digital image processing software DICe [11]. Quasi-static tests were recorded by a DINO-LITE digital microscope with high resolution (2592×1944 pixels) at 2 frames per second. High strain rate tests were recorded by high-speed camera PHOTRON SA5 at 100000 frames per second and a resolution of $512 \times 128$ pixels. The sequence of images obtained for a high strain rate test at $850^{\circ} \mathrm{C}$ is reported in Fig. 3. As it is possible to notice, no problems of oxidation were experienced even at very high temperatures (the tests were performed in air).

The different measuring systems used in the setup were synchronized with an uncertainty of 1 microsecond. The synchronization was governed by the strain-gage measuring point on the input bar. When the stress pulse was measured on it, as reported in the scheme of Fig. 1, it starts the cameras and the pulsed light system (with a properly imposed delay to have the correct level of light during the test) and switches off the heating system to avoid noise on strain-gage signals. The time interval between the moment in which the heating was switched off and the moment in which the stress pulse reaches the specimens (i.e., the test starts) is short enough (about 250 microseconds) to not induce a temperature decrease on the specimen.

\section{$4 \quad$ Experimental results}

As previously mentioned, the engineering strain was evaluated starting from the displacement time history profile obtained from digital image analysis of the recorded videos. The displacement time history was obtained by tracking the motion of some points on the specimens. For each loading condition, the displacement obtained in such a manner was compared with that obtained from the standard signal processing for Hopkinson setup. For the latter, an algorithm for the correction of the wave dispersion was applied and the wave signals were properly synchronized based on the strain-gage position along the bars. No further operation was needed, because thanks to water cooled supports the portions of bars which are heated are very small and any effects are induced on the wave propagation [10]. The comparison is reported in Fig. 4.a for a high strain rate test performed at $1000{ }^{\circ} \mathrm{C}$ on HT specimen. The velocity profiles obtained with the two methodologies differ considerably 
only in the phase in which the specimen breaks: the springback of the material produces a fast moving of the two portions of the sample in the direction of the bars with a consequent oscillation phase. Fig. 4.b shows the check of the equilibrium condition for the same test: this operation was performed for all the tests by comparing the forces on the input and output sides of the specimen. In this case, the force signals were evaluated with the standard waves equations [12].
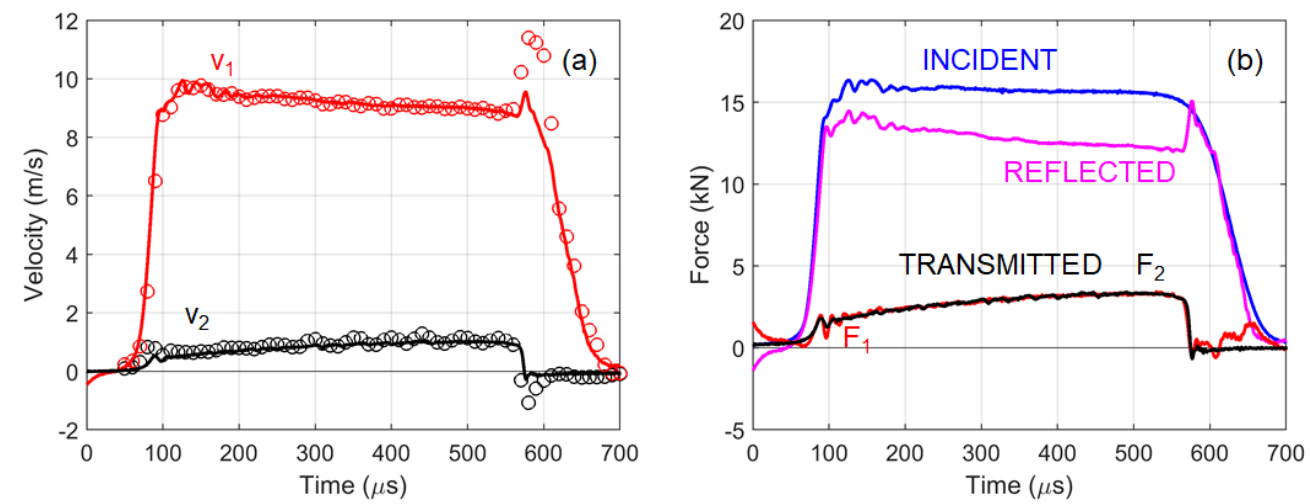

Fig. 4. High strain rate test at $1000{ }^{\circ} \mathrm{C}$ on HT specimen: (a) comparison of the input and output sides velocity time history profiles obtained by video analysis (markers) and strain-gage signal processing (solid lines); (b) check of the equilibrium condition between input and output sides of the specimen.

Fig. 5 shows the tensile response at various temperatures obtained in high strain rate tests performed on DA and HT specimens. Specimens in the as-deposited condition were not tested because as previously mentioned the interest in such a condition is negligible. The results are shown in terms of engineering stress versus engineering strain. For each loading condition only one repetition is shown for sake of clarity. For the two heat treatments, the results show the mechanical strength of the material is reduced by increasing the temperature. For temperatures up to $750{ }^{\circ} \mathrm{C}$ the reduction is limited, and the material behaviour is characterized by a low strain-hardening. In the range of temperature between 800 and $900{ }^{\circ} \mathrm{C}$ there is a significant drop of the mechanical resistance while between 900 and $1000{ }^{\circ} \mathrm{C}$ the further reduction of strength is limited.
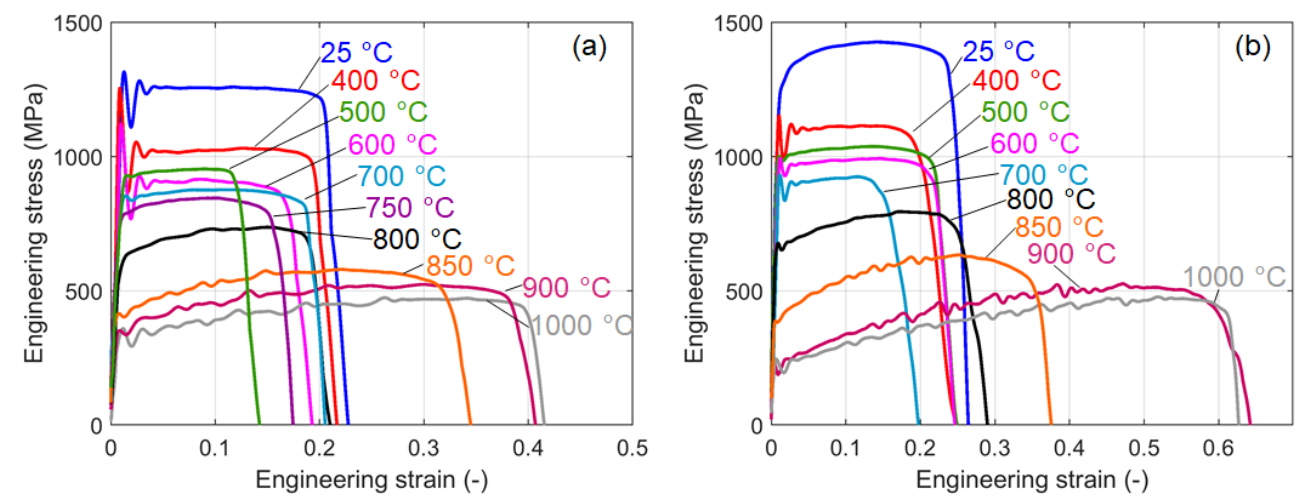

Fig. 5. Engineering stress versus engineering strain curves obtained for high strain rate tests performed on (a) DA and (b) HT specimens in the range of temperatures between 25 and $1000{ }^{\circ} \mathrm{C}$.

The serrated flow stress as observed in the diagrams of Fig. 5 for temperature higher than $800{ }^{\circ} \mathrm{C}$ could be related to Portevin-Le Chatelier (PLC) effect. As widely known PLC manifests itself as an unstable plastic flow which occurs during tensile tests of some Nickel- 
based superalloys in a certain range of temperatures and strain rates. As reported in [14] if the diffusivity of the dissolved atoms is increased by heating, the diffusion speed becomes so large that they can move to a dislocation as soon as it stops. Tearing-off and re-pinning of the dislocation alternate, causing the serrated flow curve. Another consequence of this effect is that when the strain rate is increased, the dissolved atoms may not be fast enough anymore to catch up with the dislocation and pin it. In this case, the yield strength of the material could become smaller when the strain rate increases, in contrast to the usual behaviour, and the serration of the flow curve vanishes. Consequently, the final material behaviour at a certain strain rate and temperature is a complex result of the interaction between solute atoms and dislocations and the effect of temperature is not limited to the thermal activation of dislocation obstacle overcoming but also to the solute diffusion.

Fig. 6.a shows the engineering stress versus engineering strain curves obtained for aD, DA and HT specimens at room temperature in quasi-static and high strain rate loading conditions. The strain rate sensitivity obtained covering 6 orders of magnitude was very limited or at least negligible in some cases. As previously mentioned, the final mechanical response comes as the result of the effects induced by temperature and strain rate. This implies that the effect of strain rate obtained at room temperature could not be useful to describe the material behaviour in a different temperature/strain rate condition. This was the reason for specifically performing tests at various temperatures at the strain rate of interest. In Fig. 6.b, the flow stress at 0.1 of true strain is reported as a function of the testing temperature, from which the significant decrease of the mechanical strength in the range between 800 and $900{ }^{\circ} \mathrm{C}$ as previously described can be easily appreciated as well as the stable and low downward between 400 and $750{ }^{\circ} \mathrm{C}$. In the same diagram, the results obtained for compression tests as reported in [7] are shown. Compression and tension tests were performed on the same materials with identical heat treatments ( $\mathrm{aD}, \mathrm{DA}$ and $\mathrm{HT})$, at equal nominal strain rate (1500 $\mathrm{s}^{-1}$ ) and in the same range of temperatures (from 25 to $1000^{\circ} \mathrm{C}$ ). An in-depth understanding of the results required further investigation, but Fig. 6.b reveals the asymmetry of the tensioncompression behaviour, as expected for Ni-based superalloys [15].
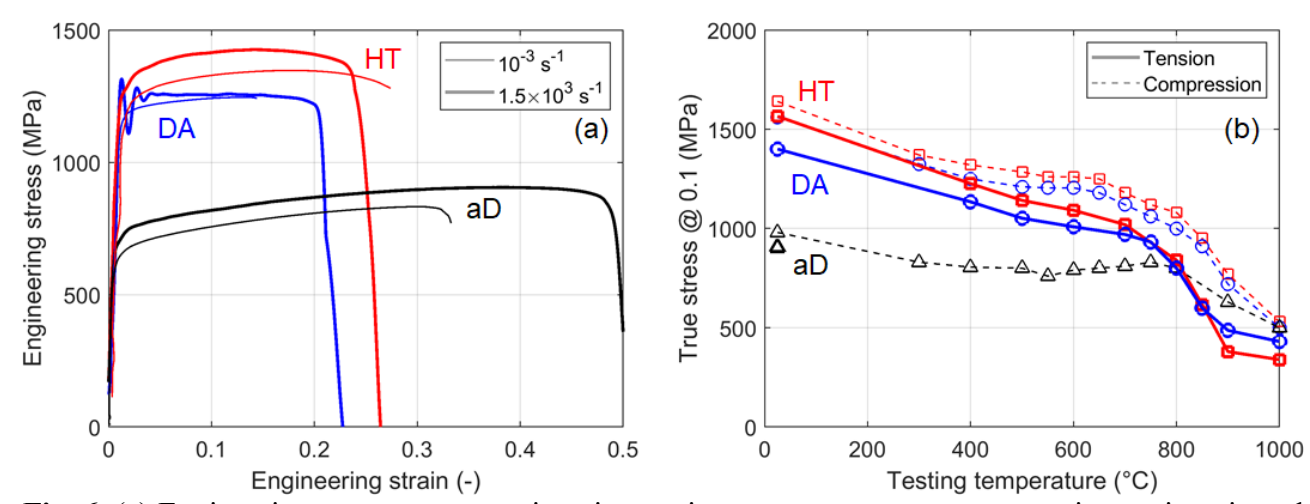

Fig. 6. (a) Engineering stress versus engineering strain curves at room temperature in quasi-static and high strain rate conditions for $\mathrm{aD}, \mathrm{DA}$ and HT specimens; (b) true stress as a function of the testing temperature at 0.1 of true strain.

\section{$5 \quad$ Conclusions}

In this study the high strain rate tensile mechanical property as a function of temperature for Inconel 718 was investigated. The need to test the material in combined high temperature and dynamic conditions came from the numerous applications in which this material is used 
for components which operate in high temperature environments being also exposed to (accidental) high dynamic mechanical stresses.

Cylindrical dog-bone specimens were machined starting from raw bars manufactured by LMD. The tensile loading direction was the deposition direction during manufacturing. Three different heat-treated conditions were analysed: as-deposited, direct-aged and aged after homogenization and solution. The aim was to investigate the increase in the mechanical strength as a function of the percentage of the strengthening phases. The as-deposited specimens were tested with the aim to highlight the need of heat treatments to improve the poor mechanical strength which was found to characterize the as-deposited condition. The nominal strain rate was $1500 \mathrm{~s}^{-1}$ and the investigated temperature varied between 25 to 1000 ${ }^{\circ} \mathrm{C}$. The tests were performed at DYNLab laboratory of Politecnico di Torino using a Hopkinson setup in direct configuration. The specimens were heated by an induction system and three different measuring systems were adopted and compared for the temperature control. The analysis of the experimental results showed that the mechanical strength slowly decreased with temperature up to $750{ }^{\circ} \mathrm{C}$ and a significant drop was found from $800{ }^{\circ} \mathrm{C}$. Finally, the comparison with previous results obtained in compression on the same materials and testing configurations revealed the tension-compression asymmetry.

\section{References}

1. Z. Li, J. Chen, S. Sui, C. Zhong, X. Lu, X. Lin, Addit. Manuf. 31 (2020)

2. R. Firoz, S.K. Basantia, N. Khutia, H.N. Bar, S. Sivaprasad, G.V.S. Murthy, J. Alloys Compd. 845 (2020)

3. S. Pratheesh Kumar, S. Elangovan, R. Mohanraj, J.R. Ramakrishna, Mater. Today: Proceedings (2021)

4. E. Hosseini, V.A. Popovich, Addit. Manuf. 30 (2019)

5. W.R. Morrow, H. Qi, I. Kim, J. Mazumder, S.J. Skerlos, J. Clean. Prod. 15, 10 (2007)

6. K. Yuan, W. Guo, P. Li, J. Wang, Y. Su, X. Lin, Y. Li, Mater. Sci. Eng. A 721 (2018)

7. K. Yuan, W. Guo, D. Li, P. Li, Y. Zhang, P. Wang, Int. J. Plast. 136 (2021)

8. M. Scapin, L. Peroni, C. Torregrosa, A. Perillo-Marcone, M. Calviani, J. dynamic behavior mater. 5 (2019)

9. M. Scapin, L. Peroni, C. Torregrosa, A. Perillo-Marcone, M. Calviani, L. Gomez Pereira, F. Léaux, M. Meyer, Int. J. Impact Eng.106 (2017)

10. M. Scapin, L. Peroni, Impact and High Strain-Rate Tests at High Temperature in Advances in Experimental Impact Mechanics, 1st Edition, Elsevier (2020)

11. D.Z. Turner, Digital Image Correlation Engine (DICe) Reference Manual, Sandia Report SAND2015-10606 O (2015)

12. M. Scapin, L. Peroni, C. Fichera, Mater. at High Temp. 31, 2 (2014)

13. P. Maj, J. Zdunek, M. Gizynski, J. Mizera, K.J. Kurzydlowski, Mater. Sci. Eng. A 619 (2014)

14. J. Roesler, H. Harders, M. Baeker, Mechanical Behaviour of Engineering Materials Metals, Ceramics, Polymers, and Composites, Springer (2007)

15. S. Ghorbanpour, M E. Alam, N.C. Ferreri, A. Kumar, B.A. McWilliams, S.C. Vogel, J. Bicknell, I.J. Beyerlein, M.Knezevic, Int. J. Plast. 125 (2020) 